\title{
Study on Gallop Early-warning System of Transmission Line Based on Real-time Weather Information
}

\author{
HUANG Junjie ${ }^{1,2, a}$, RUAN Ling ${ }^{1,2, b}$ \\ ${ }^{1}$ State Grid Hubei Electric Power Research Institute, Wuhan 430077, China \\ ${ }^{2}$ Key Laboratory of High-voltage Field-Test Technique of State Grid, Wuhan 430077, China \\ ahuangjjhwb@aliyun.com, bruanl2008@yeah.net
}

Keywords: transmission lines, gallop, early-warning, information system

\begin{abstract}
Through the analysis on the cases of the transmission line gallop over the years, the meteorological condition and the geographical location have a dramatic effect on the gallop phenomenon. And the conductor gallop can be evaluated by the thresholds of the weather factors and the degree of the terrain fluctuation. In this paper, an early-warning method for the conductor gallop on the transmission line is presented. Based on the real-time small grid weather forecast data and the geographical information, the potential gallop region can be found 3 days in advance. Applying this method, an early-warning system for the conductor gallop on the transmission line has been developed in Hubei, China. Since it was put into operation, the system has accurately predicted the gallop region and intensity many times. The practical application shows that the early-warning method has high accuracy and good practicality. Furthermore, it is helpful to develop the other early-warning systems on the transmission line, such as the icing, lighting early-warning systems.
\end{abstract}

\section{Introduction}

The overhead transmission lines are able to supply the electrical energy to the remote areas, and play an important role in the every country's development. The lines are exposed to natural winds and may therefore be subjected to the vibration or oscillation, which may impair the reliability and lifespan of the lines and its accessories. When the ice accretes on the overhead transmission lines, a wind-induced high amplitude and low frequency oscillation may be frequently observed and is named as the gallop. Due to the complex generation mechanism, difficult prevention and huge damage, the gallop is well recognized as one of the classical problems on the overhead transmission line in the world ${ }^{[1,2]}$.

In this paper, an early-warning method of the gallop, which depends on the small grid weather forecast data and the geographical information, is represented. Using the predict model of the gallop, the method is able to evaluate the potential region of the gallop in the future and give a warning in time.

\section{Mechanism of Gallop}

The gallop is a disaster composed of the meteorological condition, the geographical location and the physical property of the transmission line ${ }^{[3]}$. According to the records on the gallop phenomenon over the years in Hubei province, some results can be obtained as below.

\subsection{Ice accretion}

When the "super-cooled" rain droplets or drizzle hit the transmission lines whose temperature is below $0^{\circ} \mathrm{C}$, the ice will accrete around the lines. According to the generation mechanism, the ice accretion can be categorized by the glazed frost, the soft rime, the wet snow and so on. In the winter, the north of China has a very cold and dry climate, and there is almost nothing on the transmission lines except the snow. However, the climate of the south of China is wet and it is easy to induce the glazed frost. 
It is necessary for gallop that there are the ice accretions on the transmission lines. Under the the glazed frost, soft rime or wet snow conditions, the ice easily accretes on the surface of the conductor. When the layer thickness of the snow and ice are great, the shape of the transverse conductor section is changed from the circular into noncircular. According to the motion equation of these lines with the external force, the total damping may become the negative. The oscillations will grow and the gallop will occur.

\subsection{Wind}

A stable wind, which has a constant speed and direction, can change the shape of the ice accretion on the transmission lines under the glazed frost, the soft rime or the wet snow conditions. The transmission lines may have a better aerodynamic performance due to the asymmetrical shape of the ice accretion. In winter and early spring season of China, the strong wind is commonly caused by the intersection of the cold and warm air. When the velocity of wind is $4-20 \mathrm{~m} / \mathrm{s}$, and the angle between the wind and the line is greater than $45^{\circ}$, it is possible to gallop. if the weight of the wind whose direction is perpendicular to the line is larger, the incentive effect to uneven iced conductors is better, which is contributed for the transmission lines to accumulate energy. So the galloping of conductors can be generated easily.

\section{3 trajectory of the cold air}

Based on the meteorological data on the gallop day in Hubei over the years, the trajectory of the cold air and the result of cluster analysis have been obtained using Hybrid Single Particle Lagrangian Integrated Trajectory model (HYSPLIT). The trajectory can show the influence factors from the weather in the area such as the direction, the speed, the region of influence, and so on. The cluster analysis can group the similar influence systems in several representative influence systems and reveal the characteristics of the meteorological factor for the galloping.

From the result of the cluster analysis, there are 2 kinds of the trajectories of the dominant weather which can induce the galloping on the transmission lines in Hubei, as shown in Fig. 1.

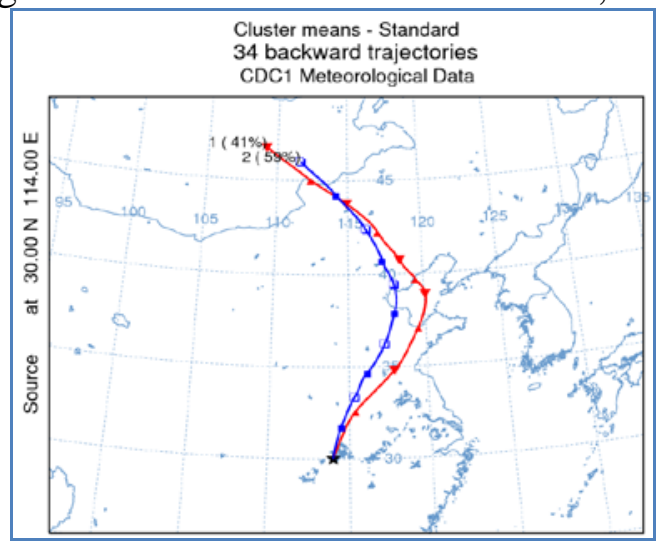

Fig. 1. The trajectories of the cold air

Through the statistical analysis of the galloping phenomenon in power grid in Hubei over the years, we can get the necessary factors for the gallop and its ranges.

\subsection{Terrain}

It is easy to make the wind with the stable speed and direction in the plain area, and the galloping is apt to occur. However, in the mountain area, the wind speed is high and the direction is random. It is difficult to induce the galloping because there is no stable force on the transmission lines ${ }^{[4]}$.

The definition of the degree of the terrain fluctuation is the difference value between the highest altitude and the lowest altitude in a selected area. DTF is a macroscopic index of the terrain features. If the terrain is flat, the value of DTF is small. On the contrary, the uneven terrain has a large value of DTF. The range of the selected area is limited by the calculation radius (CR).

\section{Predict method for gallop}

In order to predict the potential area for the gallop on the transmission lines, there are two steps that are required in the method. In the first step the trend of the gallop risk (TGR) is analyzed 
according to meteorological factors in the forecast fields. In the second step, the prediction of the gallop risk is refined by the local geographical factors ${ }^{[5,6]}$.

\subsection{Trend of gallop risk}

TGR is the possibility of the gallop occurrence in a large area. It is a rough estimate on the gallop occurrence due to the large spatial scale. However, the advantage of the trend is the short computation time in the analysis procedure, and preview the result of the analysis quickly. TGR is applied in the first step in the calculation.

TGR is calculated as follows. At firstly, using the forecast field data at the different times, a matrix of the meteorological factor is created according to the gallop risk model. Secondly, based on the update frequency and times, the matrix will be renewed twice a day. And the renewal times are at 8 p.m. and 8 a.m. in every day. If the value of the factor at the each forecast grid is in the range given by Table I, the value of the grid point is changed to a number between 0 and 1 . This value is named as the degree of predict (DP). When DP is closed to 1 , it is more possible to observe the galloping on the transmission lines in this grid point and vice versa. After all of the grid points are traversed, the analysis data of TGR with respect to time can be obtained.

\subsection{Refined prediction}

In order to refine the prediction with the local terrain factor, some extra points should be added in the grid of TGR. The position of the new points is respond to the grid of the terrain factor and the value of DP at the new points can be obtained by the interpolation.

Once the interpolation finish, the correction of TGR can be calculated following the rulers in Table I. And when the new results of each grid points have been calculated, the refined prediction of the gallop risk according to the terrain factors can be obtained. The transmission lines which have high possibility of the gallop risk can be found with the help of the buffer analysis between the value of DP and the position of the lines.

Table I. The rules for correction of terrain

\begin{tabular}{|c|c|c|c|}
\hline Trend risk & $0.7-1.0$ & & \\
\hline $\begin{array}{l}\text { Degree of fluctuation } \\
\text { Predict risk level }\end{array}$ & $\begin{array}{l}<50 \mathrm{~m} \\
\text { High }\end{array}$ & $\begin{array}{l}50-200 \mathrm{~m} \\
\text { Middle }\end{array}$ & $\begin{array}{l}>200 \mathrm{~m} \\
\text { Low }\end{array}$ \\
\hline Trend risk & $0.5-0.7$ & & \\
\hline Degree of fluctuation & $<50 \mathrm{~m}$ & $50-200 m$ & $>200 \mathrm{~m}$ \\
\hline Predict risk level & Middle & Low & None \\
\hline Trend risk & $0-0.5$ & & \\
\hline Degree of fluctuation & $<50 \mathrm{~m}$ & $50-200 m$ & $>200 \mathrm{~m}$ \\
\hline Predict risk level & Low & None & None \\
\hline
\end{tabular}

\section{Development of gallop prediction system}

Using the prediction of the galloping method in previous description, a galloping prediction system has been developed for the transmission lines. In order to satisfy the seamless connectivity between the disaster monitoring and prediction, the background data in the prediction system can be switched automatically to reliably provide the continuous service for the user.

The required data for the prediction system is derived by the meteorological department at the same time and point every day, and also the correctness and integrity of data should be checked. When the data goes through the security isolation device and enter to the intranet of power system, the product of the galloping prediction can be created and published using the geographical information and the position of the transmission lines.

Once a prediction signal occurs in the system, several warning information will send to the user within 3 days in advance.

Compared the area from the prediction system and the position of the transmission line, the transmission line which the galloping will occur can be found. It can make the prediction information with more pertinence. The warning information can be formed the product of the system and sent by the SMS or WeChat. 


\section{Application}

Under the influence of the cold air traveling from the north to south on Jan 1st, 2015, there was a large area of rain and snow in Hubei. It was a light to moderate rain and snow in northwest area, Jianghang plain, and northeast area, and a scattering of heavy snow or blizzard. Furthermore, the freezing rain with $10 \mathrm{~m} / \mathrm{s}$ north wind occurred in Jinmen, Jinzhou, and Jianghang plain. According to the meteorological data, the galloping prediction system in time released the warning information for the transmission lines.

The warning area and the gallop intensity from the prediction system had been shown in Fig.2. Compared with the monitoring data from the transmission lines, the prediction area coincided with the actual gallop points. According to the operation experiment for several years, the method is effective to predict the gallop risk on the power network.

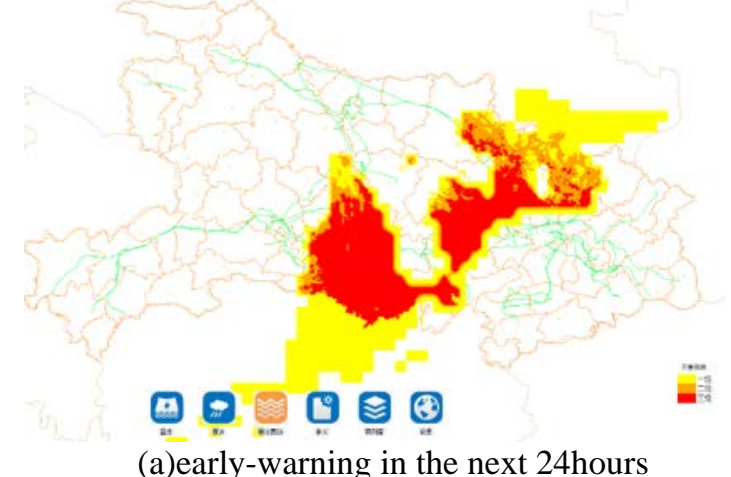

Fig. 2. results of the galloping warning and its verification

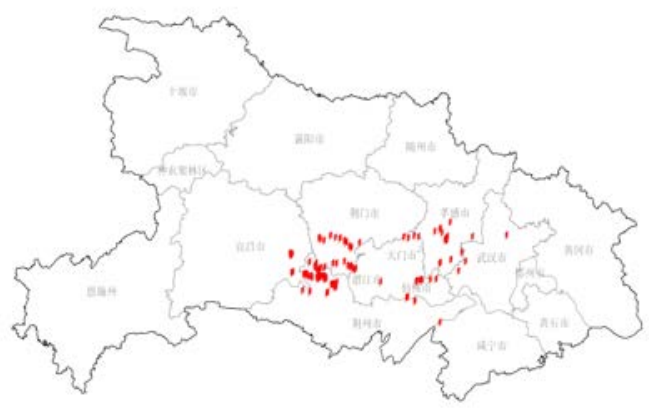

(b) actual monitored galloping points

\section{Conclusion}

The paper analyzed the mechanism of the galloping on the transmission lines and summarized the meteorological condition. Considering the effect of geographical factors, the galloping prediction method has been described. Using this method, the galloping prediction system has been developed. According to the practical verification, the method can predict the gallop area and intensity accurately. The gallop prediction system can save valuable time for the maintainer of the transmission lines. Also it is useful to help us to develop the icing or lighting predicting system.

\section{References}

[1] ZHU Kuanjun, YOU Chuanyong, ZHAO Yuanru. Study and control on galloping of transmission lines[J].Electric Power Construction,2004, 25(12):18-21.

[2] WANG Shaohua, JIANG Xingliang, SUN Caixin. Study status of conductor galloping on transmission line[J]. High Voltage Engineering, 2005, 31(10): 11-14.

[3] FAN Shexin, HE Guojin, LIAO Xiaoping, et al. Analysis of galloping mechanism of iced conductor[J]. Proceedings of the CSEE, 2006,26(14):131-133.

[4] LI Junhui, WANG Tao, ZHU Kuanjun, et al. Rendering method of galloping distribution map based on meteorological-geographical method[J]. Electric Power Construction, 2014,35(7):97-103.

[5] LI Jin, NIU Dongxiao, LIU Jinpeng, et al. Research of regional power grid crisis early warning management[J]. East China Electric Power, 2010,38(1):12-13.

[6] LIN Zhi-min, LIN Han, WEN Bu-ying. Power system reliability evaluation based on weather dependent failure models[J]. East China Electric Power, 2008,36(1):81-84. 